\title{
Experimental and Theoretical Analysis of Cabled Beams
}

\author{
Kaitlin S. Spak ${ }^{1}$ \\ Virginia Polytechnic Institute and State University, Blacksburg, VA 24060 \\ Gregory S. Agnes ${ }^{2}$ \\ Jet Propulsion Laboratory, Pasadena, CA 91011 \\ and \\ Daniel J. Inman ${ }^{3}$ \\ University of Michigan, Ann Arbor, 48109
}

\begin{abstract}
A cabled beam model is presented in which the cable is modeled as a shear beam attached to an Euler-Bernoulli beam through connections that include both linear and rotational stiffness and damping; the solution is developed through the use of the distributed transfer function method. Tension in the cable is included and a variety of damping mechanisms are incorporated. The experimental data for cabled beams is analyzed and the developed model is compared to experimental data, showing improvement over an existing distributed mass model.

rimental data, showing improvement over an existing
\end{abstract}

\section{Nomenclature}

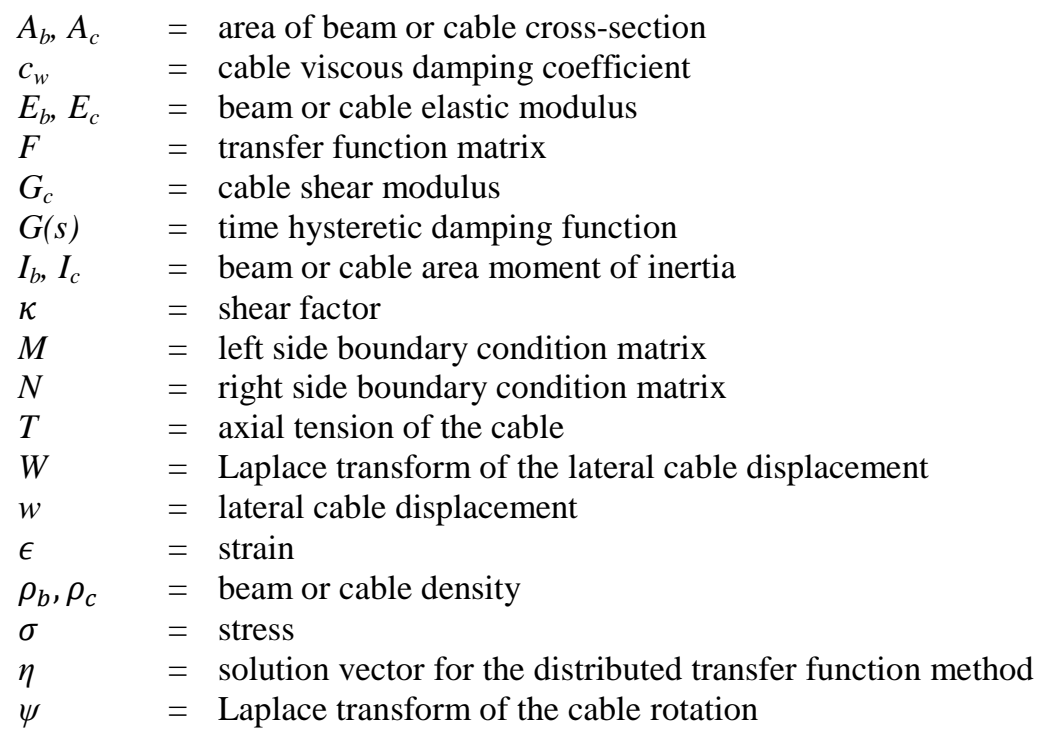

\section{Introduction}


cabled structure was significantly greater than the mass of the cables. However, the aircraft industry is seeing increasingly lightweight materials, and power and electrical requirements continue to increase in complexity. Cables are now making up a greater percentage of spacecraft mass, so the need to understand and model cable behavior for precision spacecraft control has increased. In this case, the authors are interested specifically in the dynamic response of cables used on spaceflight structures such as the one pictured in Fig. 1, although the models presented would be applicable to any cable that could be described with effective beam properties. By modeling the cable as a beam, the vibration response can be determined without considering individual friction forces between individual wires, and the bending stiffness of the cable is taken into account. This paper presents a cabled beam model developed from a beam-like equation of motion in which the cable properties are smeared to create effectively homogenous properties for the beam model. The structure to which the cable is attached is modeled as a Bernoulli-Euler beam. The distributed transfer function method is used as the solution method because it is an exact method and is well-suited to the repeating nature of cables attached to structures at multiple points.

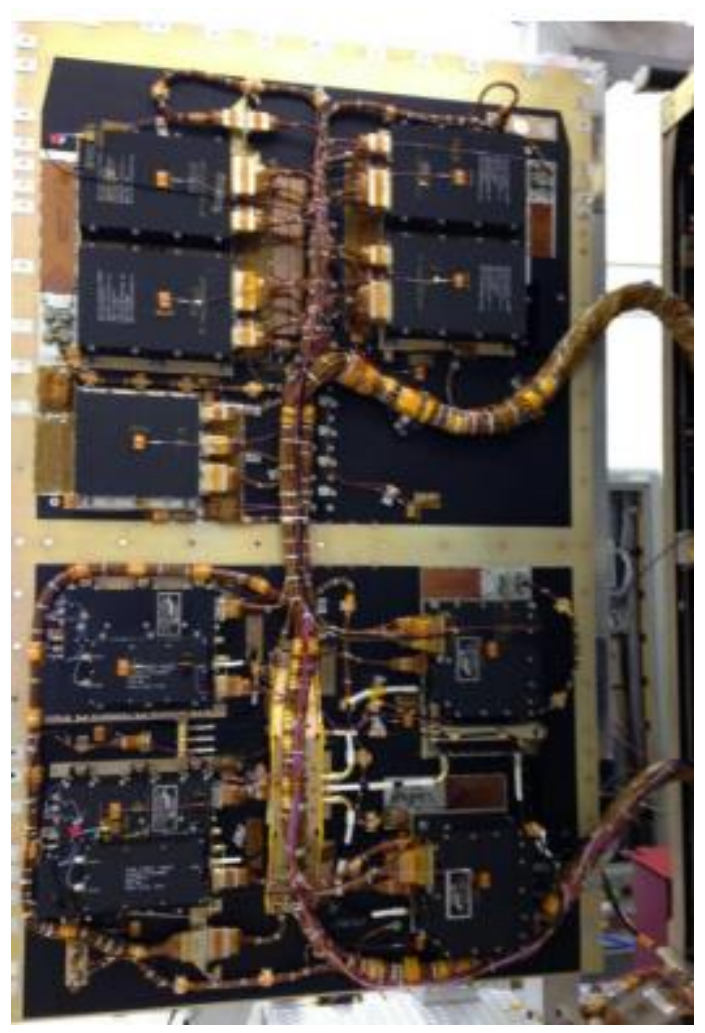

Fig. 1 Interior panel of a spacecraft showing extensive cabling.

\section{Background}

The overall structure of a cable is suited to modeling with a beam model; this has been verified by Castello and Matt [1]. Work from the Air Force Research Laboratory confirmed this and further showed that inclusion of shear effects was necessary for accurate cable modeling [2]. The bending stiffness of cables is not negligible and must be included in any cable model for accurate results. 
Cables typically have long length and small area, a combination that would normally be modeled well using Euler-Bernoulli assumptions, but cables must actually be modeled as shear beams. This is due to both the viscoelastic jacketing that undergoes shear deformation and plane sections in the cable that do not remain plane as the cable bends.

The presented cable model is the culmination of investigation that began with determining cable properties and most recently introduced a model for a cable as a shear beam.

\section{Model Development}

The cabled beam model combines the cable model presented in [3] with constraint matrices to model the attachment points and time hysteretic damping in the cable. The cables for this study have an overwrap that keeps the individual wires in the same basic configuration and maintains radial compression on the cable. As the cable bends, the wires move against each other, which alters the stiffness of the cable as a whole and is taken into account through careful calculation of the bending stiffness as a function of curvature and wire geometry. The cable is attached to a beam at multiple points by a cable tie attached to TC105 aluminum cable management tabs commonly used in the spacecraft industry.

The distributed transfer function method (DTFM) used for the system solution was developed by Yang and Tan for 1D elastic Euler Bernoulli beams [4]. The DTFM was extended for use in laminated beams, but the shear and rotary inertia effects were still neglected [5]. To use the distributed transfer function method, the Laplace transform of the equation of motion is used to populate a matrix $\boldsymbol{F}$ that can be manipulated to give information about the dynamic response of the system. This is done by rearranging the equation of motion of each part of the system to fit the form

$$
\frac{\partial}{\partial x} \eta(x, s)=F(s) \eta(x, s)+Q(x, s)
$$

where $\eta(x, s)$ is the Laplace transform of the solution vector, $F(\mathrm{~s})$ is the fundamental matrix for the system in which system properties are incorporated, and $Q(x, s)$ represents any applied forces.

Full details of the developed cable model can be found in [3], so this paper focuses on the cabled beam model. To use the DTFM, a fundamental matrix is determined based on the equation of motion. For the cable, hysteretic damping, shear, and tension terms are included in the equation of motion and the fundamental matrix is calculated as follows. For a viscoelastic material, stress is [6]:

So the moment becomes:

$$
\sigma(x, t)=E \epsilon(x, t)-\int_{0}^{t} g(t-\tau) \epsilon(x, t) d \tau
$$

$$
M=E I \psi^{\prime}-\int_{0}^{t} g(t-\tau) w^{\prime \prime} d \tau \text { OR } M=E I \psi^{\prime}-\int_{0}^{t} g(t-\tau) \psi^{\prime} d \tau
$$

Using the form in which hysteresis is applied to $w$, substitution of the moment equation into the governing equations yields:

$$
\begin{gathered}
\rho A \ddot{w}(t, x)=-\kappa A G \psi^{\prime}(t, x)+\kappa A G w^{\prime \prime}(t, x)+q(t, x) \\
E I \psi^{\prime \prime}(t, x)+\int_{0}^{t} g(t-\tau) w^{\prime \prime} d \tau-\kappa A G \psi(t, x)+\kappa A G w^{\prime}(t, x)=0
\end{gathered}
$$


The tension term is added, as well as damping terms for viscous damping and time hysteretic damping:

$$
\begin{gathered}
\rho A \ddot{w}(t, x)=-\kappa A G \psi^{\prime}(t, x)+\kappa A G w^{\prime \prime}(t, x)+c_{w} \dot{w}+q(t, x) \\
E I \psi^{\prime \prime}(t, x)+T w^{\prime}+\int_{0}^{t} g(t-\tau) w^{\prime \prime} d \tau-\kappa A G \psi(t, x)+\kappa A G w^{\prime}(t, x)=0
\end{gathered}
$$

These are combined into a single equation as follows:

$$
\rho A \ddot{w}-\frac{E I \rho}{\kappa G} \ddot{w}^{\prime \prime}+E I w^{\prime \prime \prime \prime}+\frac{E I c_{w}}{\kappa A G} \dot{w}^{\prime \prime}-\int_{0}^{t} g(t-\tau) w^{\prime \prime \prime} d \tau+-c_{w} \dot{w}+T w^{\prime \prime}=q-\frac{E I q^{\prime \prime}(t, x)}{\kappa A G}
$$

Again, the Laplace transform is taken and the equation is rearranged to match the form in which the highest derivative is set equal to the other terms.

$$
W^{\prime \prime \prime \prime}=-\frac{\rho A}{E I} s^{2} W-\frac{c_{w}}{E I} s W^{\prime}+\left(\frac{\rho}{\kappa G} s^{2}-\frac{T}{E I}-\frac{c_{w}}{\kappa A G} s\right) W^{\prime \prime}+\frac{1}{s E I} G(s) W^{\prime \prime \prime}+\frac{Q}{E I}-\frac{1}{\kappa A G} Q^{\prime \prime}
$$

This fulfills the requirement for the state space equation and we can determine that the $\boldsymbol{F}$ matrix is:

$$
F_{\text {Damped-Shear }}=\left[\begin{array}{cccc}
0 & 1 & 0 & 0 \\
0 & 0 & 1 & 0 \\
0 & 0 & 0 & 1 \\
-\frac{\rho_{c} A_{c}}{E_{c} I_{c}} s^{2} & -\frac{c_{w}}{E_{c} I_{c}} s & \frac{\rho_{c}}{\kappa G_{c}} s^{2}-\frac{T}{E_{c} I_{c}}-\frac{c_{w}}{\kappa A_{c} G_{c}} s & \frac{1}{s E_{c} I_{c}} G(s)
\end{array}\right]
$$

For the beam, the Euler-Bernoulli equation of motion is sufficient, so

$$
F_{\text {Euler-Bernoulli }}=\left[\begin{array}{cccc}
0 & 1 & 0 & 0 \\
0 & 0 & 1 & 0 \\
0 & 0 & 0 & 1 \\
-\frac{\rho_{b} A_{b}}{E_{b} I_{b}} s^{2} & 0 & 0 & 0
\end{array}\right]
$$

The fundamental matrices are manipulated in conjunction with boundary condition matrices to yield a global stiffness matrix that can be used to determine the system's natural frequencies, displacements, and mode shapes. Details of the DTFM solution method are available in [4].

The major difference between the cable model and cabled beam model is that the cabled beam model includes interaction between the cable and beam through attachment points, while the cable model is only attached to ground. For the cable model, the constraint to ground representing the TC105 attachment with stiffness is modeled as a spring connected to a very large mass representing ground. For the spring-mass constraint, Sciulli gives the equation of motion for the rigid mass and develops the constraint matrix [7]. For a beam in transverse vibration, the displacement of the node that the mass (or ground) is connected to is $u_{i}(s)=$ $\left\{\begin{array}{c}w\left(x_{i}, s\right) \\ w^{\prime}\left(x_{i}, s\right)\end{array}\right\}$ and the force is $f(s)=\left\{\begin{array}{c}M_{m}\left(x_{i}, s\right) \\ F\left(x_{i}, s\right)\end{array}\right\}$ which makes the constraint matrix term 


$$
C_{i}(s)=\left[\begin{array}{cc}
0 & 0 \\
\frac{m s^{2}(c s+k)}{m s^{2}+c s+k} & 0
\end{array}\right]
$$

The same procedure is followed to add rotational stiffness and rotational damping, but the equation of motion for the rigid mass is based on the sum of torques rather than forces, which adds an additional term to the constraint matrix to multiply the slope in the displacement vector. Thus, the complete constraint matrix for the cable attachments is

$$
C_{i}(s)=\left[\begin{array}{cc}
0 & \frac{I s^{2}\left(c_{\theta} s+k_{\theta}\right)}{I s^{2}+c_{\theta}+k_{\theta}} \\
\frac{m s^{2}(c s+k)}{m s^{2}+c s+k} & 0
\end{array}\right]
$$

where linear and rotational stiffness and damping terms are included, and $c, c_{\theta}, k$, and $k_{\theta}$ are the linear damping, rotational damping, linear stiffness, and rotational stiffness coefficients of the attachment point mechanism, which must be determined experimentally. For the cable attachments to ground, both $m$ representing the rigid mass and $I$ representing the mass inertia must be very large, at least $10^{6}$ times greater than the cable mass, to approximate the constraint condition correctly. Since the constraint matrix multiplies the displacement vector, it can be added into the global stiffness matrix when the force balance at each node representing each attachment point is determined.

For the cabled beam, the cable is connected to additional model subsystems representing the beam, rather than the ground. For this constraint matrix, the nodes of the cable and the nodes of the beam must be included. Thus, the constraint matrix must fulfill the form

$$
\left\{\begin{array}{l}
Q_{c j} \\
Q_{c l}
\end{array}\right\}=-\left[\begin{array}{ll}
C_{j j}(s) & C_{j l}(s) \\
C_{l j}(s) & C_{l l}(s)
\end{array}\right]\left\{\begin{array}{l}
u_{j}(s) \\
u_{l}(s)
\end{array}\right\}
$$

where the forces are represented by $Q$, and $j$ and $l$ are the nodes for the two subsystem nodes connected through the spring attachment. The $C$ matrices are the transfer functions that describe the attachment point with a linear and rotational spring, determined to be

$$
C=\left[\begin{array}{cc}
0 & k_{\theta}+c_{\theta} S \\
k+c s & 0
\end{array}\right]
$$

The attachment force affects both the beam and the cable, so the force balance for each node connected to an attachment point, whether it is on the beam or the cable, will have components from the attachment constraint matrix.

\section{Model Parameters}

The models presented are useful for any type of damped shear beam that may experience tension. However, to use these models for cables specifically, the cable parameters $\rho_{c}, A_{c}$, $E I_{c}$, and $G_{c}$ must be determined. Because the model assumes a homogenous cross section, these parameters must be calculated to be equivalent. This is generally done by performing dynamic 
tests and backing out the beam parameters, although current work presents a method for determining cable parameters using only cable measurements [8].

\section{Experimental Testing}

Experimental testing was performed on both cables and cabled beams to investigate the effects of the cable addition on the structure response, and to test the developed model. Four cables of varying sizes were tested; 1X7, 1X19, and 1X48 cables were small, medium, and large singlestranded cables, and $7 \times 7$ cables were multi-stranded cables made up of 6 strands of 1 X7 cables wrapped around a center $1 \mathrm{X} 7$ strand. All of the cables were machine manufactured on a planetary machine and were machine wrapped with Kapton. Figure 2 shows the suspended cabled beam, which was excited with a modal shaker and analyzed using a scanning laser vibrometer. Figure 3 shows the dense and sparse scan patterns used to collect data and determine the mode shapes of the cabled beam.

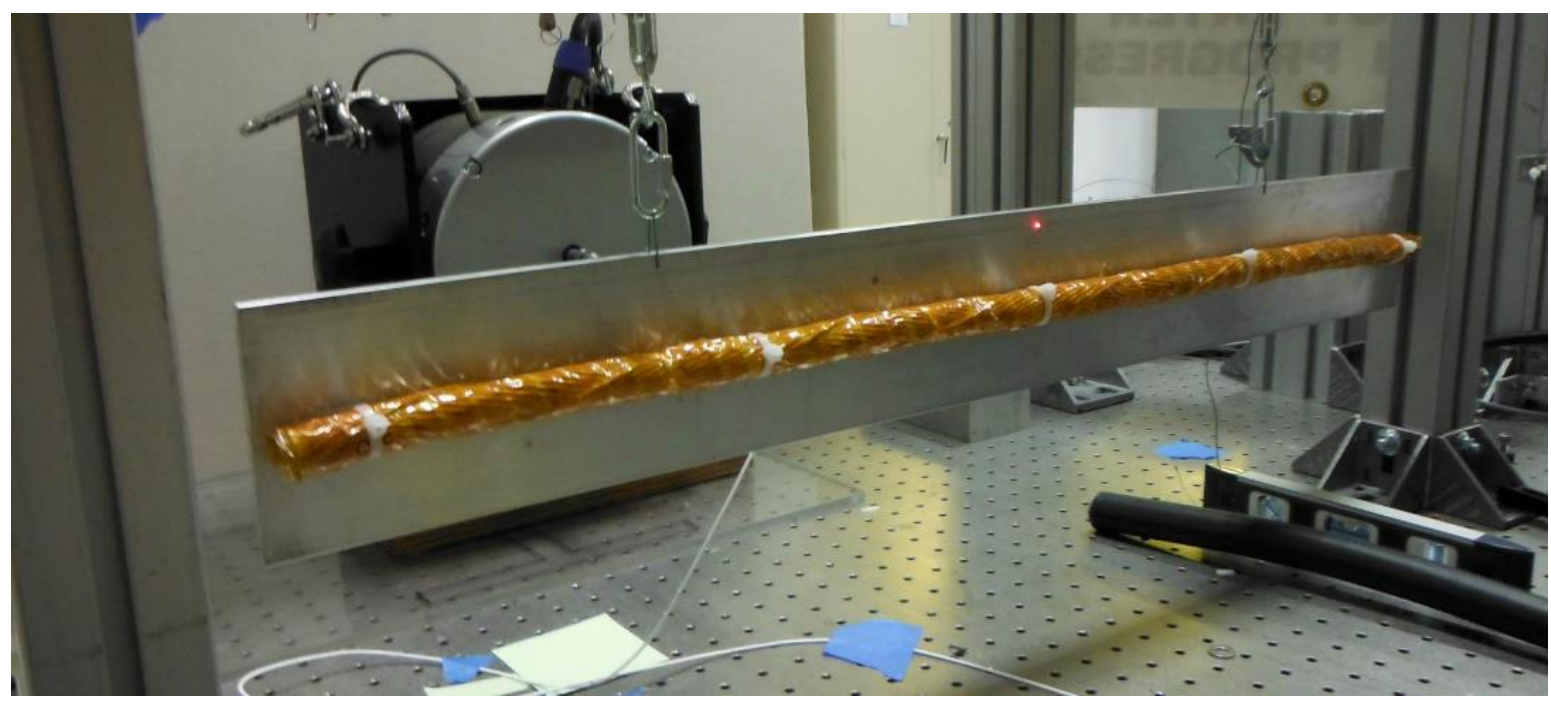

Fig. 2 Experimental testing of the $1 \mathrm{X} 48$ cabled beam, suspended to approximate free end conditions. 


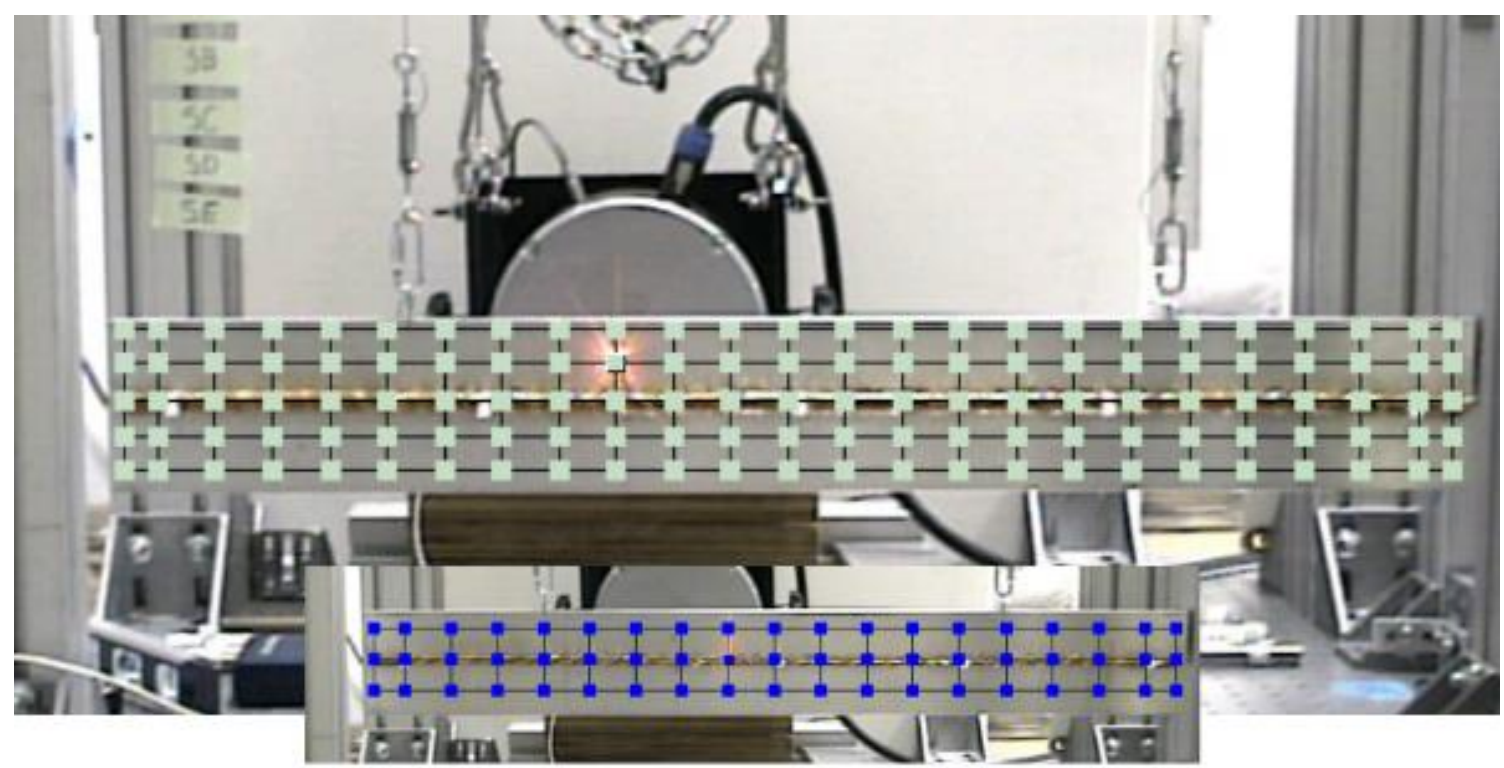

Fig. 3 Dense scan pattern and sparse scan pattern (inset) for experimental testing of cabled beams.

\section{Results}

Value of this work comes not just from the theoretical model, but also from the extensive dynamic testing performed to characterize the cabled beams. The experimental results are presented at length, followed by the comparison of the DTFM cabled beam model and distributed mass model.

\section{A. Experimental Results: Comparison of Rod and Cable on Beam}

As a valuable illustration to show the complexity of stranded cables as compared to solid materials, solid material rods of similar size, weight, and/or bending stiffness were attached to the beam and scanned with a laser vibrometer to obtain the frequency response functions. Figure 4 shows the comparison between a 1X19 cable on a beam and an Acetal rod on a beam, with the frequency response function measured at the driving point. The Acetal rod had similar mass and bending stiffness to the 1X19 cable, and the results have similarities in terms of frequency shifts and amount of damping added, but the cabled beam has more additional system frequencies than the beam with the attached rod does, particularly around the second and third bending modes. Figure 5 shows the comparison between the 1 X48 cable and a 316 stainless steel rod, which had very similar mass, but much higher bending stiffness than the cable. The results show differences between a cable and a rod attached to the beam; in each case, the beam with the solid rod attached exhibits fewer additional modes than the cabled beam does when compared to the bare beam. The solid rods also stiffen the adjacent beam at frequencies up to about $400 \mathrm{~Hz}$, shifting the natural frequencies higher despite the additional mass. The cables do not uniformly stiffen nor soften the adjacent beam, but affect each mode somewhat differently. 


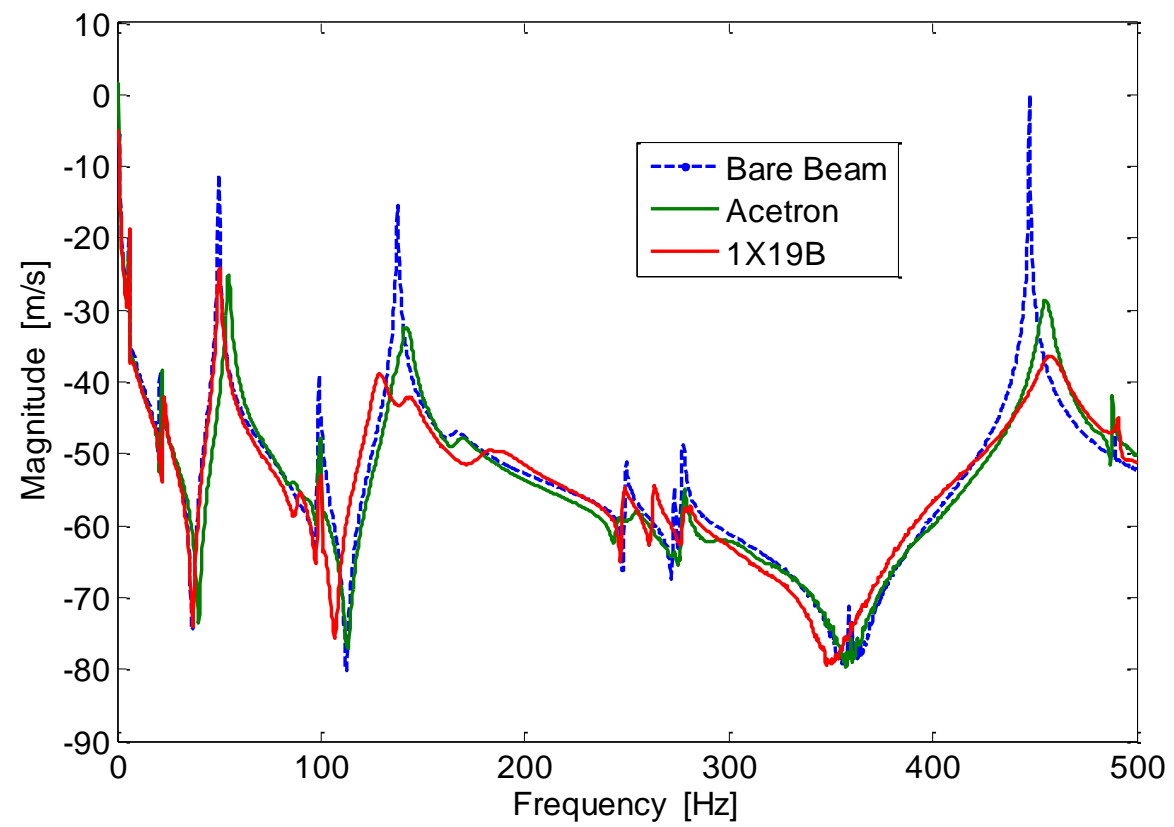

Fig. 4 Frequency response comparison between the 1X19 cable on the beam and an Acetal rod of similar mass and bending stiffness on the beam.

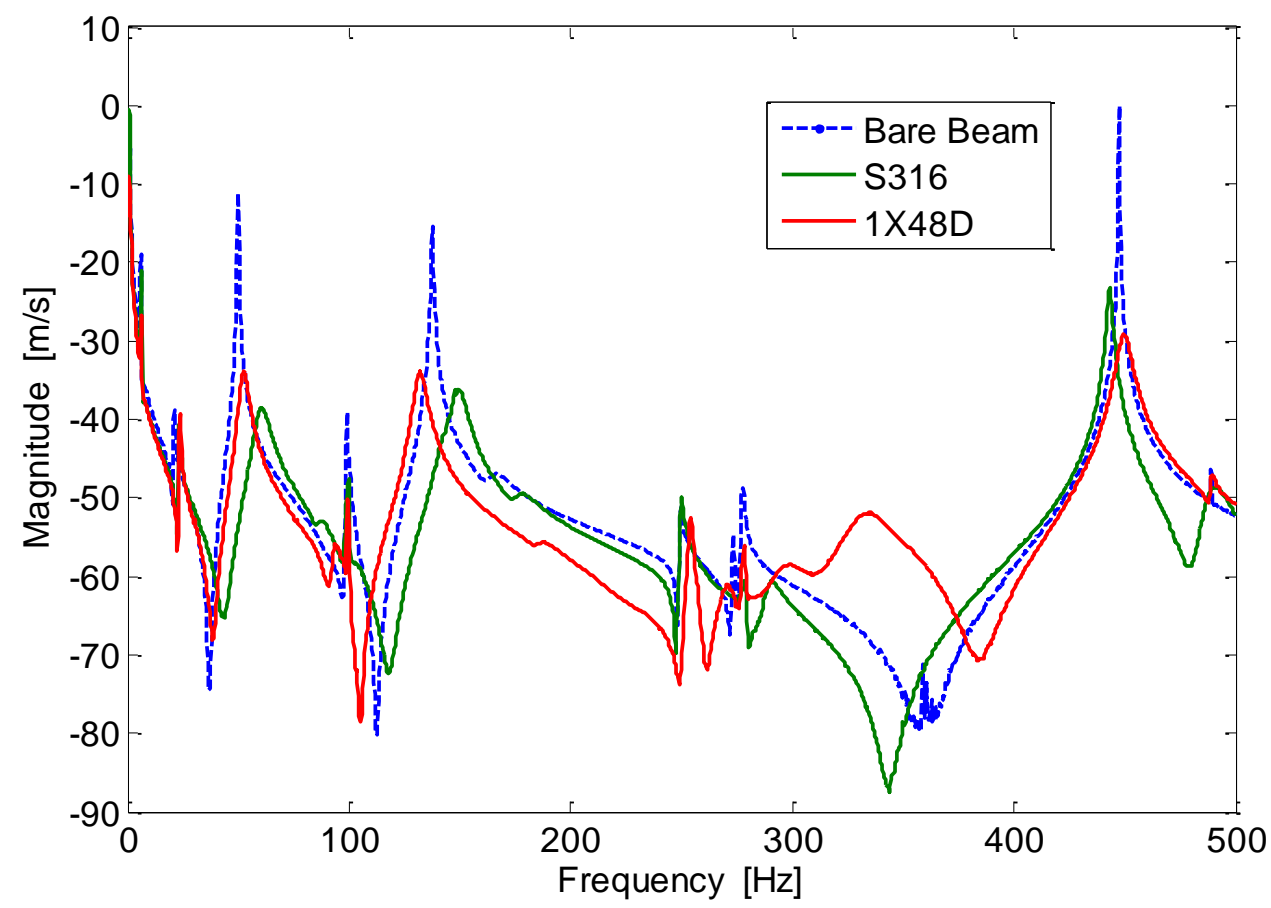

Fig. 5 Frequency response comparison between the 1X48 cable on the beam and a stainless steel 316 rod of similar mass, but much higher bending stiffness, on the beam.

\section{B. Experimental Results: Effect of Cables on Beam Dynamics}

To further the understanding of cabled structure dynamics, the experimental results from attaching different sized cables to a simple beam structure are analyzed. Figure 6 shows the frequency response function for the bare beam and for the beam with four different sized cables 
attached. It is clear that the bending modes of the bare beam still appear, but may be shifted due to the addition of the cable. The cable also causes additional modes where the cable experiences resonance; these are evident in Fig. 7 which shows the mode shapes for one of the cabled beams. Torsional modes are evident (based on observation of the mode shapes as well as the frequency response functions) for the bare beam and all cabled beams at about $250 \mathrm{~Hz}$ and $490 \mathrm{~Hz}$, which agrees with the theoretically calculated torsional modes of $246 \mathrm{~Hz}$ and $492 \mathrm{~Hz}$ for the bare beam; the addition of the cable along the middle of the beam does not affect the frequency of the torsional response. This is as expected, since the cable is mounted along the torsional axis and is only offset from the beam by a small amount. If the cable was attached above or below the center of the beam, the torsional frequencies would be affected. Different size cables do not affect the system frequency response in the same way. Additional cable modes from the 1X7 cable occur below the first bending frequency of the system, while the additional modes from the other cables add small magnitude resonance frequencies above the first system bending mode. The damping added by the cable is significant, and larger cables add more damping to the structure at most system bending modes. The response functions for the stiff 1 X48 and flexible $7 X 7$ cables show differences, although the masses of these cables were nearly identical; this shows that the bending stiffness of the cable is a necessary parameter in the cable and cabled beam models. Although the cable was attached to the beam the same way for each trial, results between cabled beam trials showed variation similar to the variation shown by the grounded cables.

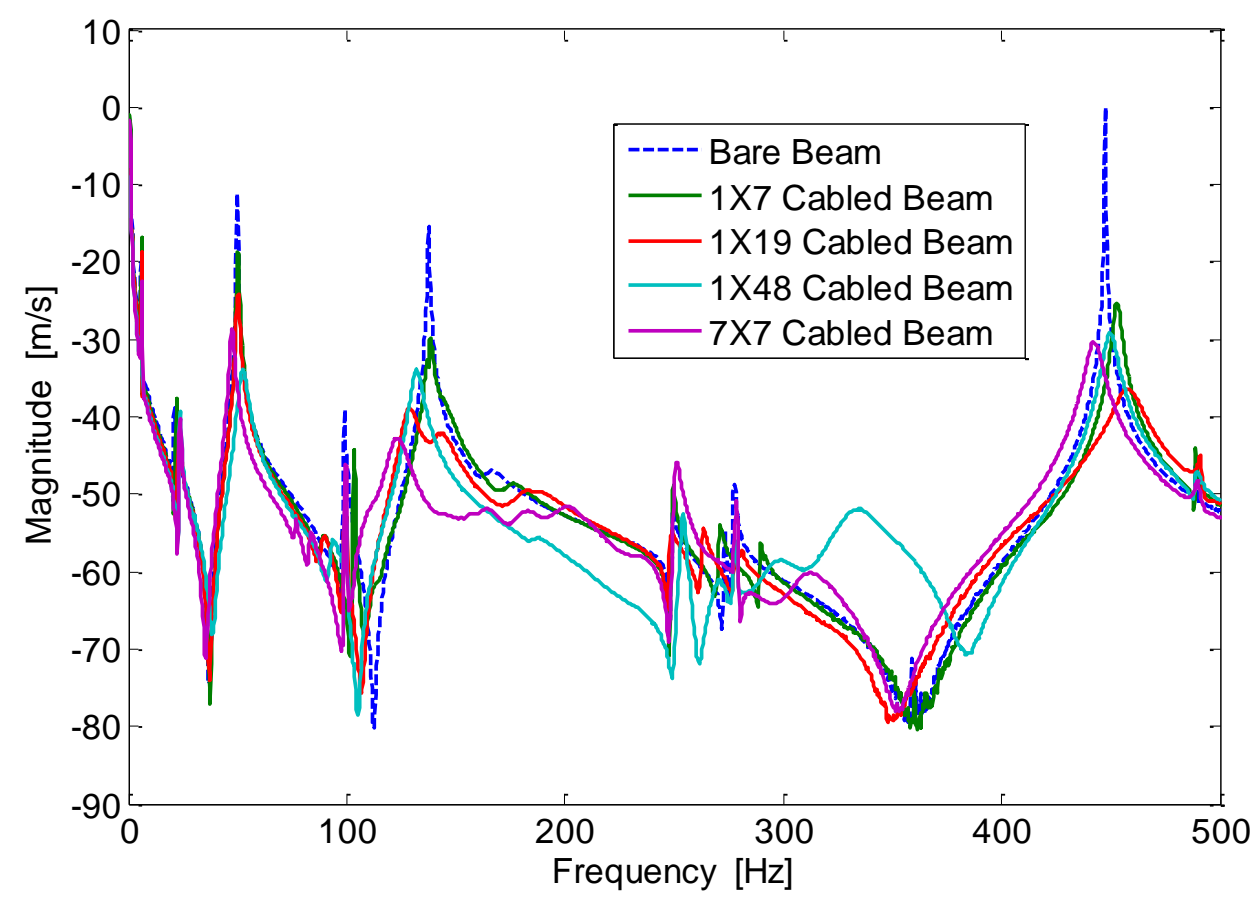

Fig. 6 Comparison of experimental data from bare beam and each cable attached to the beam, measured close to the driving point on the beam. 


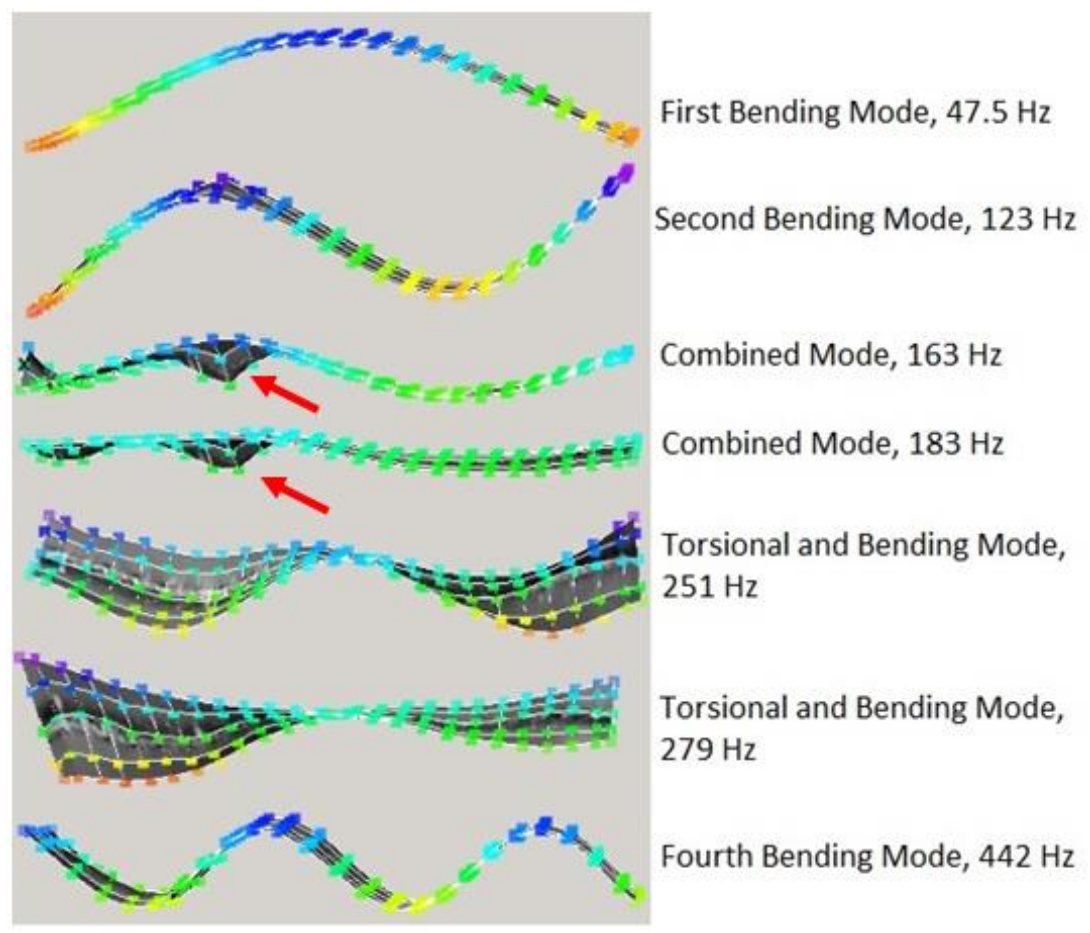

Fig. 7 Mode shapes of the 1 X48 cabled beam from the dense scan, with areas of noticeable cable deflection identified by red arrows.

\section{Theoretical Results: Comparison of Cabled Beam Model and Distributed Mass Model}

To show the value of the DTFM cabled beam model, it must show improvement when compared to existing models. The best current commonly used model is the distributed mass model; in this model, the mass of the cable is added to the beam by changing the density of the beam wherever cables are present. The distributed mass model simply shifts the natural frequencies of the bare structure; the greater the mode number, the larger the frequency decrease due to the additional mass. The distributed mass model cannot take bending stiffness into account, so the two large cables that have similar mass but different bending stiffness values are not accurately distinguished. This method is acceptable for the first one or two modes of a small mass addition, but does not capture the additional modes that arise due to the interaction between the cable and the host structure, fails to predict frequencies accurately at higher modes, and does not capture the difference between the stiff single stranded large cable and a much more flexible multi-stranded large cable. To illustrate these points, the distributed mass model is compared to the average DTFM cabled beam model and experimental data in the following figures. Figure 8 compares the experimental data and both models for the $1 \times 7$ cable. For this small cable, the differences in the model frequencies for the first two bending modes are near negligible, although the DTFM model does capture additional modes and shows closer agreement for the third and fourth bending mode frequencies. 


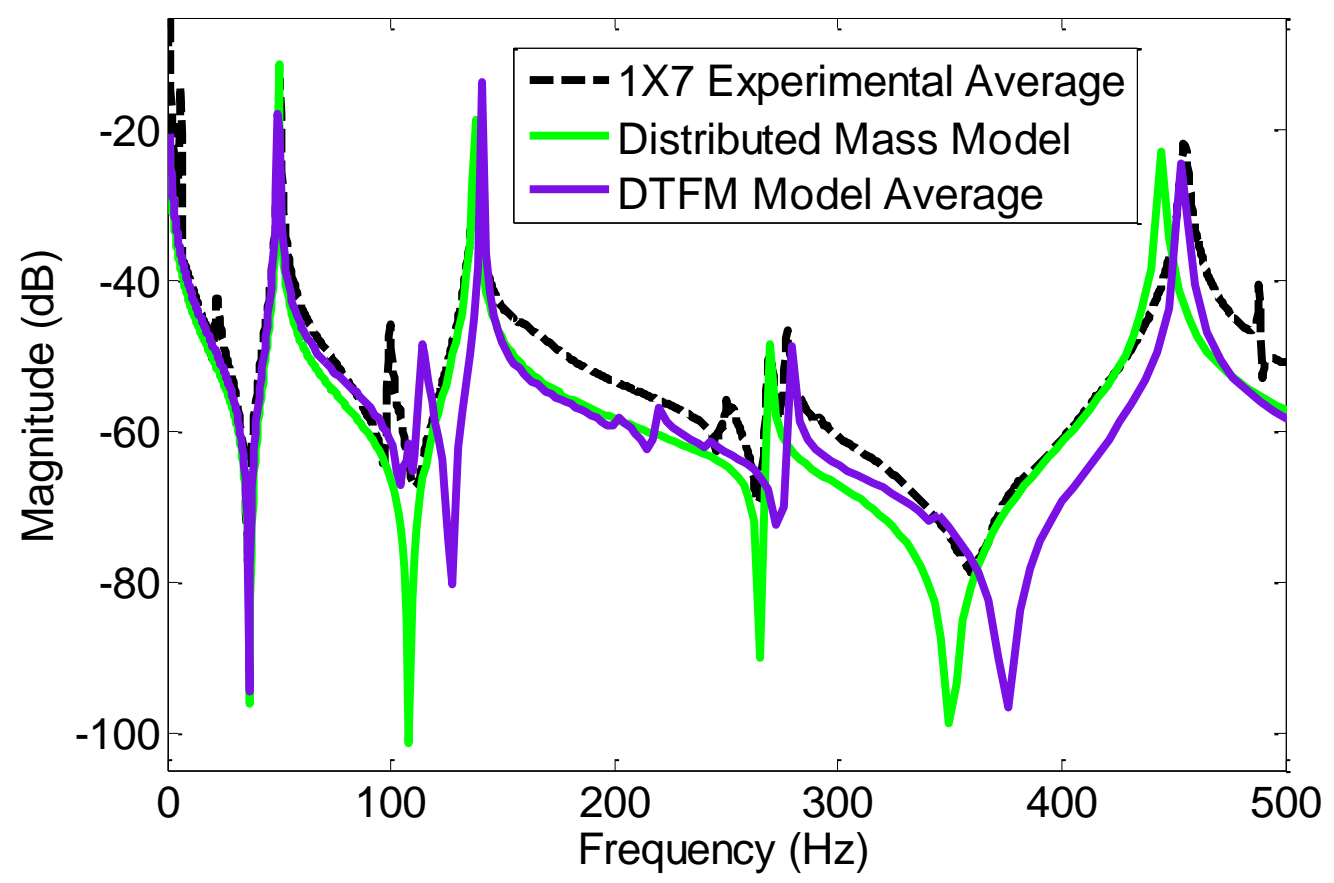

Fig. 8 Comparison of distributed mass model and DTFM cabled beam model with experimental data for 1 X7 cable.

Figure 9 and Fig. 10 compare the distributed mass model and DTFM cabled beam model for the 1X48 and 7X7 cables, respectively. Figure 9 clearly shows the addition of modes around 350 $\mathrm{Hz}$, which is not captured at all by the distributed mass model. The mode at $350 \mathrm{~Hz}$ appears to be a single mode due to trial averaging, but in reality the many cabled beam trials showed one to three modes in this area, showing good agreement with the DTFM average model. In addition, all of the bending modes are predicted more accurately by the DTFM cabled beam model for the $1 \mathrm{X} 48$ cable. The real strength of the DTFM model is shown in Figure 10, the comparison of the models and experimental data for the 7X7 model. Here, the heavy but very flexible cable has several additional modes in the $100-200 \mathrm{~Hz}$ range, and all bending frequencies are predicted closely by the the DTFM model. Amplitudes for the distributed mass model show poor agreement; amplitude response agreement for the distributed transfer function model is improved due to the addition of the more sophisticated hysteretic damping term, but further experimental analysis is required to determine the damping coefficients that would improve amplitude agreement for the additional modes due to the addition of the cable. While the 1X48 and 7X7 distributed mass models are nearly identical, the DTFM model manages to capture the differences between the cabled beams more clearly and more closely to the experimental data. 


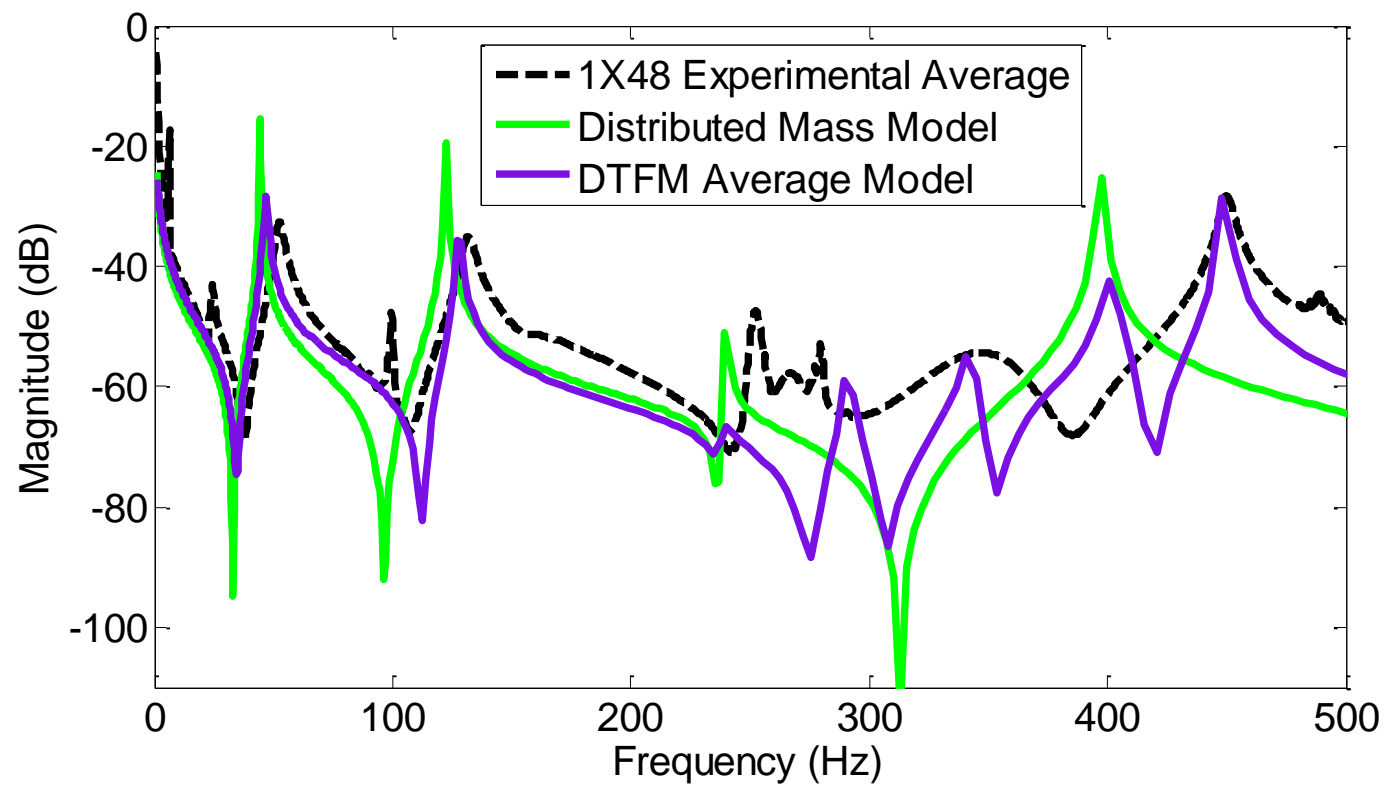

Fig. 9 Comparison of distributed mass model and DTFM cabled beam model with experimental data for 1 X48 cable.

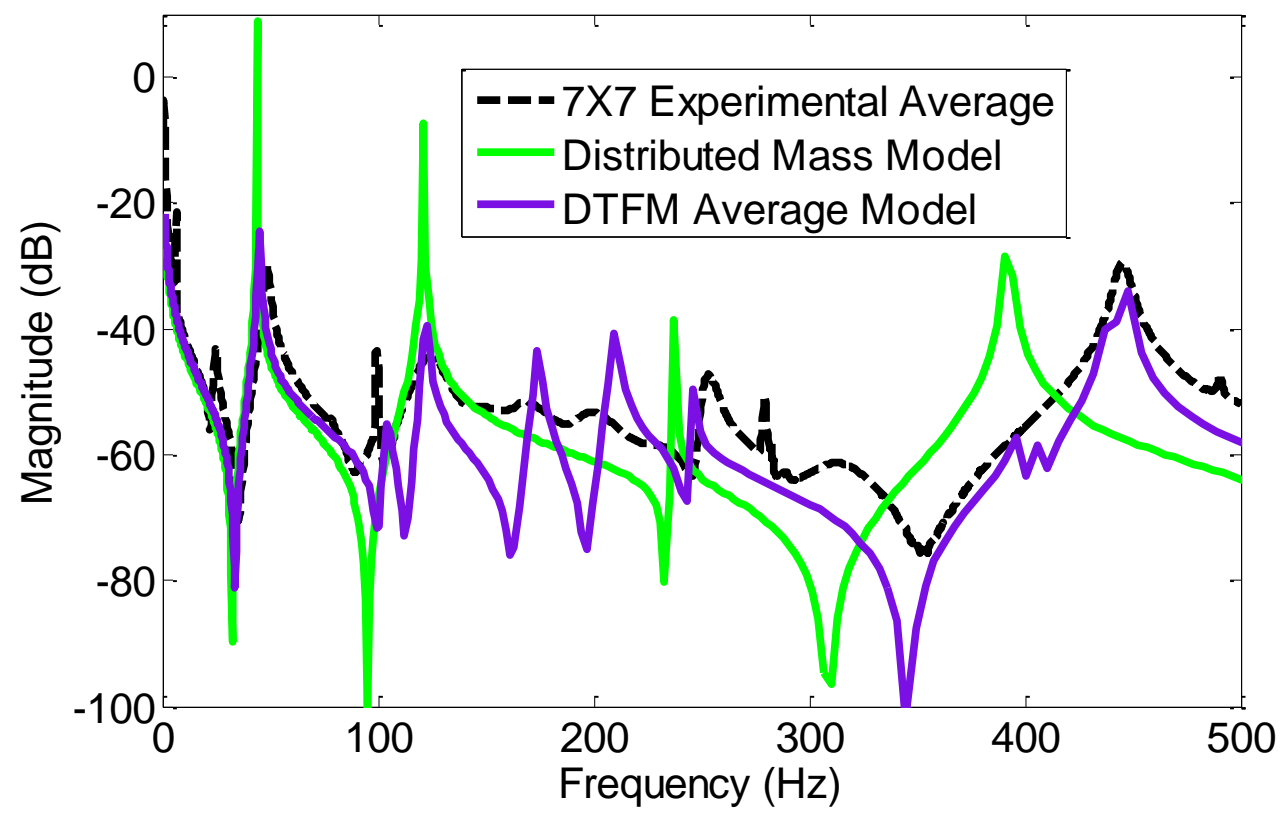

Fig. 10 Comparison of distributed mass model and DTFM cabled beam model with experimental data for 7 X7 cable.

\section{Conclusion}

The presented cabled beam model is a useful extension of the DTFM cable model. Experimental analysis of cables on a simple structure show that the structure's resonance frequencies are shifted and additional modes due to the cable attachment may be present. The frequency response predictions for the cabled beam from the DTFM model are superior to those predicted by the distributed mass model, and the DTFM model captures the additional modes 
caused by the flexible cables. The integration of this cable model into structure models will further investigation of the damping effects of structures due to cable harnesses.

\section{Acknowledgments}

The first author thanks the NASA Space Technology Research Fellowship program for generous support and the San Gabriel Valley AIAA Chapter for additional funding. Space flight cables were provided at cost by Southern California Braiding, Co. The third author gratefully acknowledges the support of AFOSR Grant number FA9550-10-1-0427 monitored by Dr. David Stargel. This research was carried out at the Jet Propulsion Laboratory, California Institute of Technology, under a contract with the National Aeronautics and Space Administration.

\section{References}

${ }^{1}$ Castello D. A., and Matt C. F. T., "A Validation Metrics Based Model Calibration Applied on Stranded Cables," Journal of the Brazilian Society of Mechanical Scientists and Engineers, Vol. 33, No. 4, 2011, pp. 417-427.

${ }^{2}$ Goodding, J. C., Ardelean, E. V., Babuska, V., Robertson, L. M., and Lane, S. A., "Experimental Techniques and Structural Parameter Estimation Studies of Spacecraft Cables," Journal of Spacecraft and Rockets, Vol. 48, No. 6, 2011, pp. 942-957.

${ }^{3}$ Spak, K.S., Agnes, G.S., and Inman, D.J., "Inclusion of Shear Effects, Tension, and Damping in a DTF Beam Model for Cable Modeling," 55th AIAA/ASME/ASCE/AHS/ASC Structures, Structural Dynamics, and Materials Conference, National Harbor, MD, 2014, AIAA 2014-0491.

${ }^{4}$ Yang B., "Distributed Transfer Function Analysis of Complex Distributed Parameter Systems," Journal of Applied Mechanics, Vol. 61, No. 3, 1994, pp. 84-92.

${ }^{5}$ Majeed, A.M., Al-Ajmi, M., and Benjeddou, A., "Semi-analytical Free-Vibration Analysis of Piezoelectric Adaptive Beams using the Distributed Transfer Function Approach," Structural Control and Health Monitoring, Vol. 18, 2011, pp. 723-736.

${ }^{6}$ Inman, D.J., "Vibration Analysis of Viscoelastic Beams by Separation of Variables and Modal Analysis," Mechanics Research Communications, Vol. 16, No. 4, 1989, pp. 213-218.

${ }^{7}$ Sciulli, D., "Dynamics and Control for Vibration Isolation Design," Dissertation, Virginia Tech, 1997.

${ }^{8}$ Spak, K.S., Agnes, G.S., Inman D.J., "Parameters for Modeling Stranded Cables as Structural Beams," Experimental Mechanics, 2014, doi: 10.1007/s11340-014-9941-8. 